\title{
Exposure to virtual social stimuli modulates subjective pain reports
}

\author{
Jacob M Vigil PhD, Daniel Torres BS, Alexander Wolff, Katy Hughes
}

\begin{abstract}
JM Vigil, D Torres, A Wolff, K Hughes. Exposure to virtual social stimuli modulates subjective pain reports. Pain Res Manag 2014;19(4):e103-e108.
\end{abstract}

BACKGROUND: Contextual factors, including the gender of researchers, influence experimental and patient pain reports. It is currently not known how social stimuli influence pain percepts, nor which types of sensory modalities of communication, such as auditory, visual or olfactory cues associated with person perception and gender processing, produce these effects.

OBJECTIVES: To determine whether exposure to two forms of social stimuli (audio and visual) from a virtual male or female stranger modulates cold pressor task (CPT) pain reports.

METHODS: Participants with similar demographic characteristics conducted a CPT in solitude, without the physical presence of an experimenter or another person. During the CPT, participants were exposed to the voice and image of a virtual male or female stranger. The voices had analogous vocal prosody, provided no semantic information (spoken in a foreign language) and differed only in pitch; the images depicted a middleage male or female health care practitioner.

RESULTS: Male participants, but not females, showed higher CPT pain intensity when they were exposed to the female stimuli compared with the male stimuli. Follow-up analyses showed that the association between the social stimuli and variability in pain sensitivity was not moderated by individual differences in subjective (eg, self-image) or objective measurements of one's physical stature.

DISCUSSION: The findings show that exposure to virtual, gender-based auditory and visual social stimuli influences exogenous pain sensitivity. CONCLUSION: Further research on how contextual factors, such as the vocal properties of health care examiners and exposure to background voices, may influence momentary pain perception is necessary for creating more standardized methods for measuring patient pain reports in clinical settings.

Key Words: Experimental pain; Gender; Pain assessment; Psychology; Social determinants of

E xperimental pain tests provide essential information for understanding individual differences in pain sensitivities and clinical pain experiences (1-3). The efficacy of these experiments is contingent on the reliability of the methods used and the ability to control contextual factors that can influence pain measurements. Basic contextual factors, such as the gender of experimenters and other individuals in the immediate social context, have been shown to influence subjective pain reports (4-8). The majority of previous pain experiments have been subject to these potential confounds. What is not known is which sensory modalities of communication (eg, auditory, visual, olfactory cues) associated with person perception and gender processing influence experimental pain reporting. In the present study, we targeted two basic modalities of gender processing that may influence cold pressor task (CPT) pain performance - auditory and visual processing of a virtual male and a virtual female stranger stimulus while controlling for the potential influence of laboratory personnel characteristics.

\section{L'exposition à des stimuli sociaux virtuels module les déclarations de douleur subjective}

\begin{abstract}
HISTORIQUE : Des facteurs contextuels, y compris le sexe des chercheurs, influent sur les déclarations de douleur en milieu expérimental et auprès des patients. On ne sait pas quelle est l'influence des stimuli sociaux sur la perception de la douleur ni quels types de modalités sensorielles liées à la communication, telles que les indices auditifs, visuels ou olfactifs associés aux perceptions individuelles et au traitement du sexe de l'individu, produisent ces effets.
\end{abstract}

OBJECTIFS : Déterminer si l'exposition à deux formes de stimuli sociaux (audio et visuel) provenant d'un étranger virtuel de sexe masculin ou féminin module les déclarations de douleur à un test au froid.

MÉTHODOLOGIE : Les participants partageant des caractéristiques démographiques ont effectué un test au froid eux-mêmes, hors de la présence physique d'un expérimentateur ou d'une autre personne. Pendant ce test, ils étaient exposés à la voix et à l'image d'un étranger virtuel de sexe masculin ou féminin. Les voix avaient une prosodie vocale similaire, ne fournissaient pas d'information sémantique (propos dans une langue étrangère) et différaient seulement sur le plan du ton. Les images montraient un dispensateur ou une dispensatrice de soins d'âge mûr.

RÉSULTATS : Les participants de sexe masculin, mais pas ceux de sexe féminin, présentaient une intensité de douleur plus élevée à un test au froid lorsqu'ils étaient exposés aux stimuli d'une femme qu'à ceux d'un homme. Les analyses de suivi ont révélé que l'association entre les stimuli sociaux et la variabilité de la sensibilité à la douleur n'était pas modérée par les différences individuelles des mesures subjectives (p. ex., perception de soi) ou objectives de la stature physique personnelle.

EXPOSÉ : D'après les résultats, l'exposition à des stimuli audio et visuels virtuels, fondés sur le sexe, influe sur la sensibilité à la douleur exogène. CONCLUSION : Des recherches plus approfondies s'imposent sur l'influence des facteurs contextuels, tels que les propriétés vocales des examinateurs et l'exposition à des voix en arrière-plan, sur la perception de la douleur momentanée, afin de créer des méthodes plus standardisées de mesurer les déclarations de douleur des patients en milieu clinique.

Several studies show that interactions with experimenters differing in basic categories (eg, gender, authority role) and even the passive presence of others in the immediate social context can influence subjective and autonomic pain responses $(6,9,10)$. For example, some research has shown that individuals sometimes demonstrate heightened exogenous pain sensitivity when they are in the presence of a female researcher or peer $(4,6,11)$. Other studies have found that, for men only, passive interactions with female researchers, health examiners or female strangers during an experimental pain task (cold pressor, ischemic) can reduce pain sensitivity $(5,8,12)$. Thus, it appears that, at least among women, the presence of female strangers tends to produce a hyperalgesic effect, whereas in men, the presence of females may result in both amplified as well as reduced pain sensitivities.

These findings have been explained from a social signalling (evolutionary psychology) perspective, which is based on the prediction that females evolved the behavioural heuristic to express higher levels of pain-reaction behaviours (eg, hyperalgesia) and pain-empathizing 
behaviours than males in general, as part of a broader expressive style characterized by the heightened demonstration of trustworthiness cues $(8,13-18)$. According to this perspective, people should implicitly perceive women as sources of solicitude, consolation and logistical assistance, which would have coevolved with the behavioural heuristic for individuals, especially women, to experience heightened pain percepts (hyperalgesia) in the presence of other women. However, women can also serve as prospective reproductive partners for males, and men tend to use empowerment gestures and demonstrated prowess to attract potential mates (19-21). Therefore, it makes sense that the biological costs/benefit fitness tradeoffs associated with demonstrating heightened or lessened vulnerability, such as pain behaviours, in the presence of a woman should, theoretically, be contingent on implicit social expectations of the woman and other situational factors such as the momentary state of physical prowess versus debilitation of the individual experiencing pain $(8,16,17,22-24)$. This reasoning leads to the hypothesis that men are more likely to experience hyperalgesia in the physical presence of a woman when the man is significantly debilitated (eg, experiences from clinical, chronic and/or endogenous pain) and, thus, when the ability to demonstrate physical prowess is compromised (12). Men should instead experience hypoalgesia to impress newly acquainted women (via fitness cues) when the man is relatively capable and, hence, when he is not experiencing debilitating pain, as is the case with most experimental discomfort tasks.

No extant research has attempted to discriminate how social stimuli in the immediate context influences momentary pain percepts, such as whether the effect is the result of basic visual, auditory, olfactory or perhaps other (eg, thermal detection) person-perception sensory processing. A reasonable starting point to address this major gap in the literature is to examine how virtual social stimuli, such as audio and visual cues, associated with basic biological categories, such as gender, may affect momentary pain sensitivity, while controlling for the potential influence of examiner characteristics. To the best of our knowledge, no study published to date has investigated the role of human vocal properties on momentary pain perception, and previous studies that have examined visual properties of social stimuli (eg, photographs of family members, facial expressions of emotion, body images) were confounded by the role of examiner characteristics (2426). Historically, most experimental pain studies have not controlled for the influence of examiner characteristics on subjective pain reports, either methodologically or statistically. Research examining how sounds may influence health in general has shown that the noise volume of medical equipment (eg, in a hospital room) can affect patient outcomes and treatment satisfaction (27-32), as well as experimental pain performance (33). The one study that examined the role of vocal properties of the health care providers themselves found that the physician's vocal tone was reciprocally related to patient-provided health reports (34). Other research has indicated that treatment satisfaction is influenced by the gender of health examiners and patients, such that patients (and particularly males) examined by younger, female physicians reported less satisfaction (35).

In the current experiment, we examined whether exposure to nonsemantic (content-free) male or female human voices spoken in an obscure foreign language, and visual depictions of a male or female health care provider influences CPT pain reports in healthy young adults with similar ethnic backgrounds and demographic characteristics. The present study uniquely used a CPT protocol that enabledparticipants to complete the discomfort task entirely on their own, without the actual physical presence of a researcher during the task itself; this enables measurement of the exclusive influence of the discrete virtual stimuli (rather than other social contextual factors) on pain performance. Based on the social-signalling perspective of momentary pain $(16,17,22,23)$ and previous research described above $(5,8)$, we hypothesized that exposure to female vocal/visual stimuli (but not male stimuli) would be associated with hyperalgesia among women, and hypoalgesia among men. Secondary analyses examined whether subjective (self-rated face and body appearance) and objective measurements (height and shoulder circumference) of physical stature moderated any potential association between exposure to the social stimuli and variability in pain sensitivity. These analyses were prompted by research showing that morphological attributes, such as height, influence person perception, including subjective impressions of dominant trait attributes in other people (36).

\section{METHODS}

\section{Participants}

The protocol was approved by the University of New Mexico's Institutional Review Board (Albuquerque, New Mexico) and two forms of written consent were obtained from all participants. The first consent form described the general experimental protocol, and the second described the CPT in more detail. Participants included a convenience sample of young adults (American citizens) who were self-described as having a predominantly Hispanic/Latin-American ethnic background. Limiting the participants to a particular ethnic group within a narrow age range helps to control for individual variability in cultural experiences, which may influence a person's perception. Participants who self-identified contraindication(s) to the CPT were excluded from the study. Contraindications included any illness related to a cardiovascular disorder (eg, high blood pressure, heart disease or dysrhythmia); history of fainting or seizures; history of frostbite; having an open cut, sore or bone fracture on the limb to be immersed in water; or a history of Reynaud's phenomenon (abnormal discoloration of the skin with exposure to heat and cold). The sample consisted of 120 adults (18 to 25 years of age; mean $[ \pm \mathrm{SD}$ ] age $19.5 \pm 1.5$ years; $33 \%$ men). Three different experimental conditions were used in the present study, and participants were randomly assigned to the three conditions. These were: exposure to the virtual male stimuli $(n=35)$; exposure to the virtual female stimuli $(n=41)$; and a control condition in which no social stimuli was used $(n=43)$. A $\chi^{2}$ test of independence showed that the sex ratio of participants was not statistically different across the experimental conditions $(\mathrm{P}>0.10)$.

\section{Protocols}

Participants were assisted through the experimental protocol by one of 15 research assistants; seven self-identified males, seven self-identified females, and one self-identified male-to-female transgender researcher (50\% Hispanic/Latin-American, 50\% non-Hispanic white). A $\chi^{2}$ test of independence showed that gender and ethnicity of the researchers was not statistically different across the experimental conditions (all $\mathrm{P}>0.10$ ). The researchers followed a script for every phase of the experiment to minimize the possible influence of interpersonal factors (eg, duration of conversations) associated with researcher characteristics. Following the informed consent procedure, participants were measured for height and shoulder circumference; this took 3 min to 5 min. Participants were then escorted to an assessment room, where they were left alone to complete a demographic questionnaire and to view a video that provided instructions for performing the CPT without a researcher present. The video provided directions for using the cold pressor apparatus and indicated various pain ratings. The survey and instructional video took approximately $30 \mathrm{~min}$ to complete.

Once participants finished watching the instructional video, they were led into the room with the cold pressor apparatus. The cold pressor room was fitted with a video camera for viewing the participants from a remote location, a video/audio monitor for presenting the social stimuli, a cold pressor apparatus and a laptop programmed with userinterfaced pain assessment software. The computer program was used to electronically measure participants' self-indicated pain intensity ratings at $30 \mathrm{~s}$ into the CPT, as well as the time latency for when the participant first felt a painful sensation (pain threshold), and when they chose to discontinue the task because they could no longer tolerate the pain (pain tolerance), as described below. The participants were instructed to begin the task once they heard/viewed the audio/ visual stimuli (programmed to initiate $15 \mathrm{~s}$ after the researcher left the CPT room). The individual participant then performed the CPT 
without a researcher present. The researcher observed the participant through a live video feed from the next room to ensure adherence to the cold pressor procedure. This innovative protocol enabled researchers to collect CPT data without being physically present during the $\mathrm{CPT}$, which has been shown to influence experimental pain sensitivity $(5,6,8)$. Following the CPT, individuals were debriefed.

\section{Questionnaires}

A basic questionnaire created by the authors' group included questions regarding major demographic characteristics such as sex, age, ethnicity and subjective ratings of one's appearance. The ratings consisted of two items asking participants to describe their feelings about the appearance of their face and body, respectively (coded from 1 to 5, from having strong negative feelings to having strong positive feelings).

\section{CPT}

Cold pressor apparatus: Participants were seated in a chair between the pressor apparatus (left side) and the laptop computer (right side) in a small room $(2.0 \mathrm{~m} \times 2.5 \mathrm{~m})$. The mechanical CPT device was an Isotemp 6200R28 (Fisher Scientific, USA) refrigerated bath circulator (reservoir size $27.9 \mathrm{~cm} \times 16.5 \mathrm{~cm} \times 22.4 \mathrm{~cm}$ ). The machine circulates the water automatically and maintains a consistent water temperature by dual heating and cooling actions. The water temperature was set to $5^{\circ} \mathrm{C}$ (known to produce a range of pain tolerance levels with only minimal ceiling effects [37]). Small differences in water temperature $\left(2^{\circ} \mathrm{C}\right)$ can have significant effects on pain sensitivity measures (38), and all the participants in the current study experienced water temperatures within $0.5^{\circ} \mathrm{C}$ of one another.

Cold pressor procedures: The pain assessment program (on the laptop computer) displayed an initial screen with the general CPT instructions. The researcher verbally reiterated the instructions by describing that when participants choose to both begin (on initiation of the social stimuli) and end the task (at maximum pain tolerance) they were to perform two simultaneous actions. To begin the task (and initiate the pain assessment program), participants were instructed to first indicate their baseline (premanipulation) pain severity on a standard visual analogue scale ( 0 to 10 , ranging from no pain to worst pain imaginable), while simultaneously submerging their left hand into the cold water to a marked line on the wrist (one inch above the wrist joint). To end the task, participants were instructed to indicate this preference electronically by clicking on a corresponding icon on the computer screen while simultaneous lifting their hand out of the cold pressor apparatus. Participants were also instructed to immediately indicate when they first experienced a pain sensation (pain threshold) and to indicate their felt pain intensity $30 \mathrm{~s}$ into the CPT (automatically prompted by an audio notification and illumination of the visual analogue scale).

Once the participants indicated their understanding of the instructions, they were fitted with a finger pulsometer to monitor their heart rate during the CPT. Finally, the researcher reminded the participant that they would be recorded, and that they should begin the task once they heard/viewed the audio/video stimuli projecting from a 17 inch $(43.2 \mathrm{~cm})$ monitor placed six feet $(1.83 \mathrm{~m})$ in front of the participant. The researcher then left the cold pressor room and closed the door. The stimuli were programmed to begin $15 \mathrm{~s}$ after the researcher closed the door of the experimental room. The procedure was observed on a video monitor from a remote location, and the researcher returned to the experimental room to debrief the participant once they retracted their hand from the water or after the maximum duration of $5 \mathrm{~min}$ (the participants were not informed of this time limit). Following debriefing, participants were asked to rest for $5 \mathrm{~min}$ to ensure they no longer felt any physical discomfort from involvement in the study and that their heart rate had returned to resting.

\section{Virtual social stimuli}

In the experimental conditions, a video track depicting a male or female voice was merged with a static image of a middle-age, Caucasian individual in a laboratory/physician coat with a concordant gender. To avoid the confounds of prosodic differences in male and female speech styles, such as inflection, tone, speed, resonance and pronunciation, the same audio clip was used to create both the male and female voice tracks. The voice tracks were created from a single audio file of a male narrator reciting biblical studies in a Finnish dialect. This language was chosen because of its obscurity and to avoid the influence of speech content; a post-task survey verified that none of the participants recognized the spoken content from the audio tracks. An open-source commercial software (Audacity ${ }^{\circledR}$ ) was used to create a female voice from the vocal track by adjusting the pitch of the original file. During the pitchshifting process, the audio file's duration was extended to 200\% playback speed, then the voice's intonation was adjusted from $A$ sharp ( $A \#)$ to $D$ before returning the audio track to its original duration. Another opensource software program (Kdenlive) was then used to merge the two audio clips (the male voice and the female voice) with their respective photographs to create the video files. The videos were each rendered with dimensions of $1440 \times 1080$ (1080p) using the common H.264/ MPEG-4 Part 10 or Advanced Video Coding video compression format, with a frame rate of $25 \mathrm{fps}$ at an average 29 kilobits per second; the low video bit rate is sufficient for the static images. The videos were formatted in compliance with the International Organization for Standardization/International Electrotechnical Commission MPEG-4 Part 14 (MP4). Audio was encoded using the common MPEG 4 Audio (M4A) format. Each file contained $15 \mathrm{~s}$ of black noise, which allowed the experimenter to leave the room before the audio/visual stimulus began. The duration of the videos was $6 \mathrm{~min} 30 \mathrm{~s}$. The control condition was created by replacing the video of the social stimuli with a video of a static image of clouds; no audio stimulus was used for this condition.

\section{Data analyses}

The pain scores included the participant's pain threshold, pain tolerance (measured in seconds postsubmersion), and the pain intensity score $30 \mathrm{~s}$ into the CPT (participants whose pain tolerance was $<30 \mathrm{~s}$ [13\% of sample] were given a pain intensity score of 8 of 10). Higher intensity scores and lower threshold and tolerance scores are interpreted as indicating greater CPT pain sensitivity (reverse effect sizes of threshold and tolerance scores reflect greater pain sensitivity). ANCOVAs were used to examine the separate effects of the gender of the individual and the gender of virtual stimuli on the pain scores; participant's baseline (premanipulation) pain scores were entered as a covariate. Independent-samples $t$ tests were used to compare group differences and effect sizes pertaining to group comparisons were estimated with Cohen's d (mean difference/mean SD [39]).

\section{Social stimuli and pain performance}

An ANCOVA was performed for each of the three pain scores (pain threshold, pain tolerance and pain intensity) using the Experimental Condition (male stimuli, female stimuli or controls), Participant Gender (males coded 0, females coded 1), and the Condition $\times$ Participant Gender interaction terms as predictor variables (entering baseline pain scores as a covariate). A trend for a significant Condition $\times$ Participant Gender interaction term emerged for pain tolerance $(\mathrm{F}[2,110]=2.67 ; \mathrm{P}=0.074)$, and the interaction terms were significant for pain threshold $(F[2,108]=3.05 ; \mathrm{P}=0.051)$ and for pain intensity $(F[2,99]=4.75 ; P=0.011)$. Follow-up analyses examining the effect of the experimental manipulation on the pain threshold and pain intensity scores separately for men and women showed a significant main effect for pain intensity in men only $(F[2,33]=3.95 ; \mathrm{P}=0.029)$; the effect of the experimental condition on pain intensity among women and the effect of the experiment on pain threshold in both sexes were not significant (all $\mathrm{P}>0.10$ ).

The mean pain intensity scores for men and women across the experimental conditions are shown in Figure 1. In men only, exposure to the male and female social stimuli produced opposing effects on pain intensity, although the scores from these conditions were not 


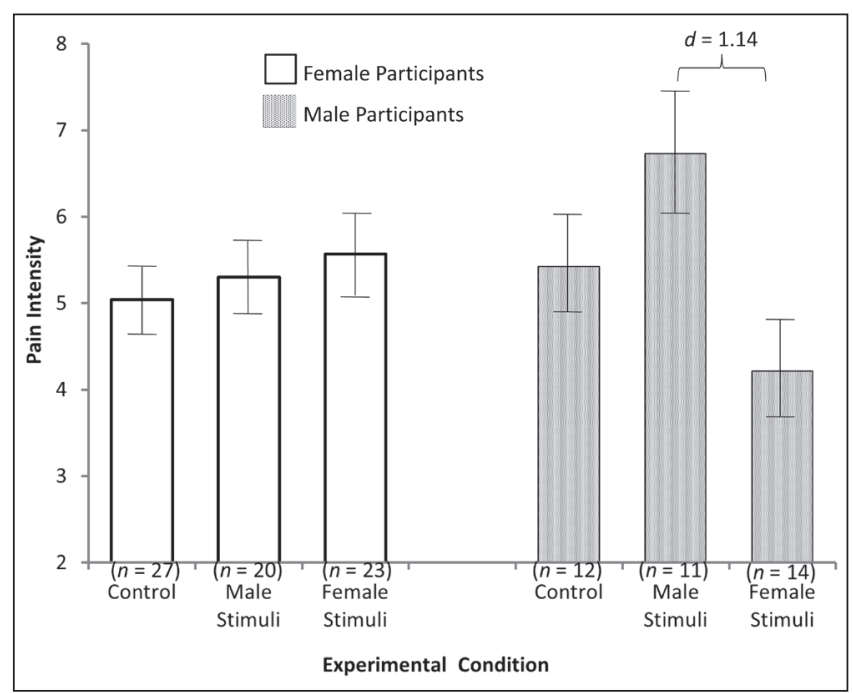

Figure 1) Group differences in pain intensity for men and women exposed to virtual male or female social stimuli or the control condition. Bars represent SEM

statistically different from the control condition (all $\mathrm{P}>0.10$ ). The group difference in the pain intensity scores for men exposed to male versus female stimuli was significant $(\mathrm{t}[23]=2.85 ; \mathrm{P}=0.009)$.

\section{Individual differences in social influences}

Partial correlations (controlling for baseline pain) in the male participants were significant between the pain intensity scores, and self-rated facial appearance $(r=0.34 ; \mathrm{P}=0.050)$ and body build $(\mathrm{r}=0.37 ; \mathrm{P}=0.034)$. The correlations between pain intensity and height and shoulder circumference were not significant (all $\mathrm{P}>0.10$ ). Separate regressions examining whether self-rated facial appearance or body build moderated the relationship between exposure to male versus female social stimuli (dichotomously coded) and pain intensity showed nonsignificant (Stimuli $\times$ Face, Stimuli $\times$ Body build) interaction terms for both equations (all $\mathrm{P}>0.10$ ). Collectively, these findings show that individual differences in objective body proportions and subjective ratings of physical appearance do not influence how men experience differential pain intensity with exposure to virtual male or female social stimuli.

\section{DISCUSSION}

The present study uniquely provides support that gender-based audio and visual stimuli of virtual strangers influence experimental pain sensitivity, and these effects differ for men and women. Specifically, and similar to previous studies investigating the impact of audience effects on experimental pain tasks $(6,8)$, we found that exposure to the voice and image of a virtual female stimulus resulted in lower mean pain intensity than exposure to a virtual male stimulus, and this effect was limited to men. Our other hypothesis (that women would instead experience heightened pain sensitivity with exposure to female stimuli) was not supported. Follow-up analyses examining individual differences in these effects showed that the men's subjective ratings of their own physical appearance were associated with pain sensitivity; however, neither subjective nor objective measurements of one's appearance moderated the effect of the social stimuli on pain intensity. Thus, the findings replicate other studies showing that the perceived presence of other people in the immediate context influences subjective pain reporting during standard experimental and clinical pain assessments $(8,12)$. Collectively, this research suggests that: young men in particular tend to experience hypoalgesia in the immediate and simulated presence of female strangers; men tend to experience hyperalgesia in the presence of male stimuli; and these effects are most pronounced when the individual experiencing pain is experiencing relatively low levels of pain intensity, such as that which occurs during conventional experimental discomfort tasks. Rather, men and women have been shown to report higher pain levels to female versus male examiners when the individual experiencing pain is more debilitated and, hence, experiencing clinically relevant (ie, high) levels of pain (12). The findings, therefore, support the obvious need for basic scientists and clinicians to develop more standardized protocols for measuring discrete pain experiences.

The observed patterns are generally interpretable from the socialsignalling perspective that: variability in pain sensitivity and associated pain gestures operate (in part) at the behavioural level for demonstrating basic trait impressions of vulnerability versus empowerment to other people; and implicit social expectations of individuals in the immediate social context modulate the perception and subsequent expression of felt pain $(8,16,17,22-24)$. Human females likely evolved a greater sensitivity to express higher levels of both pain-suffering and pain-empathizing behaviours than males on average $(8,12,16-18)$, and both men and women have been shown to experience higher pain intensity in the presence of women, particularly when the individual experiencing pain is experiencing relatively high (eg, clinical) levels of pain (12). This phenomenon makes sense based on the general hypothesis that individuals should heuristically express higher levels of behaviours that connote the impression of vulnerability to the types of social agents who are most likely to provide solicitous responses to the person in need $(16,17)$. However, when men in particular are experiencing relative milder and more escapable forms of pain sensations, such as during experimental discomfort tasks, they may implicitly process female strangers as prospective reproductive partners (rather than sources of solicitude). Under these conditions, men may demonstrate reduced ability to detect pain in ways that potentiate the natural tendency to demonstrate physical prowess to potential mates. Nonetheless, at the most basic empirical level, the current findings are consistent with the broader literature showing that (often unavoidable) contextual and methodological factors associated with subjective pain reporting confound the reliable measurement of pain experiences.

The findings are also intriguing for understanding how specific modalities of sensory processing can influence momentary pain percepts. Although the current study involved the use of both (simultaneous) audio and visual input, it is likely that the audio stimuli took up more attentional resources than the visual stimuli. This is because the auditory stimuli could not be easily inhibited (eg, via gaze-aversion) and because it provided more continuous and dynamic (eg, novel, phasic) information than the visual stimuli throughout the CPT. It is, therefore, likely that the auditory stimuli, in and of itself, provided the greatest contribution to the present findings. Likewise, because the auditory stimuli were content free (spoken in an unfamiliar language) and congruent in every aspect of vocal prosody (eg, inflection, duration and pauses of speech), except for the pitch of the voices, it is possible that sensory processing of pitch detection (eg, sound frequency) may have uniquely driven the observed effects.

Regardless of this specific hypothesis, the current protocol was able to show that exposure to virtual gender cues can induce hypoalgesia in some people, and that physical interactions with and exposure to the actual physical properties (eg, pheromones, touch and heat radiation) of another person are not necessary to modulate the intensity of felt pain. Such possibilities have implications for the eventual design of novel pain treatment options that may operate via passive or active means by simulating specific forms of social stimuli (eg, gender cues) in ways that naturally alleviate pain. For example, it may be possible that modifications to contextual factors associated with the décor of clinical environments (visual depictions of women, music or commentary with female vocalists) and devices that simulate gender-based social information (pitch- and prosody-specific, low-emission auditory devices) may result in analgesia for some patients. Strategic use of different types of vocal properties, such as sound frequency, may also be useful for moderating the outcomes of therapeutic techniques that rely on vocal communication. At the very least, the current findings are clinically important for understanding how the tone of 
health examiners' voices and exposure to background sounds (eg, in a hospital room) can influence patient pain reporting. Contextual factors, including 'audience effects', contribute to current sources of measurement error in most clinical settings (12), and staff training in vocal communication styles and/or use of computer interfaces should improve the ability to reliably assess patient pain experiences across timepoints, patient cohorts and health care settings. In theory, nearly every form of patient (subjective) symptom reporting can be affected by contextual factors such as characteristics of people in the immediate audience. As in pain perception (12), these effects may be influenced by various factors such as social expectations of the audience members, and/or the patient's physical or mental condition.

Despite these implications, the present initial study had some limitations that should be addressed in future research. First, the sample size was small, potentially hindering ability to detect significant effects in some of the analyses. Methodologically, we did not control for handedness, which has been shown to influence CPT measurements (40). Also, reactions to CPT may not predict reactions to other types of exogenous or endogenous discomfort sensations, and results from the relatively distinct sample (young, Hispanic adults) may not be generalizable to different demographic groups. Another potential limitation was the use of single-item scales to measure individual differences in self-construals, which may influence how people report pain intensity in the presence of social stimuli. Use of both auditory and visual cues confounded the ability to examine how each respective sensory input may have influenced the results. Future studies that can systematically isolate not only different modalities of communication, but also specific properties of the social signals themselves (eg, associated with gender perception) are needed to disentangle the influence of social contextual experiences on subjective pain reporting. Studies that can parse varying elements of pain processing, such as attentional modulation, autonomic functioning and neuroendocrine activation, will be particularly useful for contributing to this line of research. Finally, although we attempted to control the potential influence of observer effects, it is still possible that individuals responded to the

\section{REFERENCES}

1. Clauw DJ, Williams D, Lauerman W, et al. Pain sensitivity as a correlate of clinical status in individuals with chronic low back pain. Funct Restor 1999;24:2035-41.

2. Kim H, Neubert JK, Rowan JS, Brahim JS, Iadrola MJ, Dionee RA. Comparison of experimental and acute clinical pain responses in humans as pain phenotypes. J Pain 2005;5:377-84.

3. Carvalho B, Zheng M, Aiono-Le Tagalona L. Evaluation of experimental pain tests to predict labour pain and epidural analgesic consumption. Br J Anaesth 2013;110:600-6.

4. Gijsbergs K, Nicholson F. Experimental pain thresholds influenced by sex of experimenter. Percept Mot Skills 2005;101:803-7.

5. Levine FM, De Simone LL. The effects of experimenter gender on pain report in male and female subjects. Pain 1991;44:69-72.

6. Kállai I, Barke A, Voss U. The effects of experimenter characteristics on pain reports in women and men. Pain 2004;112:142-7.

7. Rowell LN, Mechlin B, Ji E, Addamo M, Girdler S. Asians differ from non-Hispanic Whites in experimental pain sensitivity. Eur J Pain 2011;15:764-71.

8. Vigil JM, Coulombe P. Biological sex and social setting affects pain intensity and observational coding of other people's pain behaviors. Pain 2011;152:2125-30.

9. Brown JL, Sheffield D, Leary MR, Robinson ME. Social support and experimental pain. Psychosom Med 2003;65:276-83.

10. Kleck RE, Vaughan RC, Cartwright-Smith J, Vaughan KB, Colby CZ, Lanzetta JT. Effects of being observed on expressive, subjective, and physiological responses to painful stimuli. J Pers Soc Psych 1976;34:1211-8.

11. McClelland LE, McCubbin JA. Social influence and pain response in women and men. J Behav Med 2008;31:413-20.

12. Vigil JM, Alcock, J. Tough guys or sensitive guys? Disentangling the role of examiner sex on patient pain reports. Pain Res Manag 2014;19:e9-e12. remote presence of the experimenter (in another room) in ways that confounded the ability to examine our proposed hypotheses. Our laboratory has shown that even minimal procedural interactions (eg, processing consent procedures and explaining the research protocol) with laboratory personnel before a discomfort task can influence experimental pain reports (41). However, given the competing and attention-demanding nature of the experimental stimuli used in the current study (ie, continuous exposure to human voices), it is likely that the influence of experimenter characteristics played a secondary role in participants' pain performance.

\section{SUMMARY}

The current findings extend previous research on sex differences in social situational factors that influence pain perception by showing exposure to auditory and visual stimuli of a virtual female stranger, but not stimuli of a male stranger, modulates CPT pain sensitivity in healthy young men. Better understanding of how social experiences modulate pain perception in vivo and in vitro is, therefore, important for designing more standardized protocols for reliably measuring subjective pain reports. Although it is not yet clear how these findings may be transformed into the design of novel pain treatment options at the present time, they provide an important first step for isolating certain domains of social stimuli (ie, auditory and visual) that can induce hypoalgesic effects in some people. At the very least, these findings suggest that contextual factors, such as basic vocal properties of health care examiners' voices, can influence momentary pain perception. Further research is, therefore, necessary for understanding how and when these effects may occur to create more standardized techniques for measuring pain experiences in research and medical environments.

ACKNOWLEDGEMENTS: The authors have no financial or other conflicts of interest associated with this work to declare. The first author has access to the data and takes responsibility for the integrity of the data and the accuracy of the data analysis.
13. Geary DC, Byrd-Craven J, Hoard MK, Vigil J, Numtee C. Evolution and development of boys' social behavior. Dev Rev 2003;23:444-70.

14. Vigil JM. Asymmetries in the friendship preferences and social styles of men and women. Hum Nat 2007;18:143-61.

15. Vigil JM. Sex differences in affect behaviors, desired social responses, and accuracy at understanding the social desires of other people. Evol Psych 2008;6:506-22.

16. Vigil JM. A socio-relational framework of sex differences in the expression of emotion. Behav Brain Sci 2009;32:375-90.

17. Vigil JM. The socio-relational framework of expressive behaviors as an integrative psychological paradigm. Behav Brain Sci 2009;32:408-28.

18. Vigil JM. Current states of opinion and future directions on the epidemiology of sex differences in human pain. Pain Res Manag 2011;16:317-9.

19. Buss DM. Evolutionary Psychology: The New Science of the Mind, 4th edn. Needham Heights: Allyn \& Bacon, 2011.

20. Geary DC. Male, Female: The Evolution of Human Sex Differences, 2nd edn. Washington, DC: American Psychological Association, 2010.

21. Geary DC, Vigil J, Byrd-Craven J. The evolution of human mate choice. J Sex Res 2004;41:27-42.

22. Cano A, Williams A. Social interaction in pain: Reinforcing pain behaviors or building intimacy? Pain 2010;149:9-11.

23. Craig KD. The social communication model of pain. Can Psychol 2009;50:22-32.

24. Schiavenato M, Craig KD. Pain assessment as a social transaction beyond the "gold standard." Clin J Pain 2010;26:667-76.

25. Master SL, Eisenberger, NI, Taylor SE, Naliboff BD, Shirinyan D, Lieberman MD. A picture's worth: Partner photographs reduce experimentally induced pain. Psychological Science 2009;20:1316-8.

26. Senkowski D, Kautz J, Hauck M, Zimmermnn R, Engel AK. Emotional facial expression modulate pain-induced Beta and 
Gamma oscillations in sensorimotor cortex.

J Neurosci 2011;31:14542-50.

27. Johnson AN. Neonatal response to control of noise inside the incubator. Pediatr Nurs 2001:27:600-5.

28. Slevin M, Farrington N, Duffy G, Daly L, Murphy JF. Altering the NICU and measuring infants' responses. Acta Paediatrica 2000;89:577-81.

29. Zahr LK, de Traversay J. Premature infant responses to noise reduction by earmuffs: Effects on behavioral and physiologic measures. J Perinatol 1995;15:448-55.

30. Baker CF. Discomfort to environmental noise: Heart rate responses of SICU patients. Crit Care Nurs Q 1992;15:75-90.

31. Novaes MA, Aronovich A, Ferraz MB, Knobel E. Stressors in ICU: Patients' evaluation. Intensive Care Med 1997;23:1282-5.

32. Topf M, Thompson S. Interactive relationships between hospital patients' noise-induced stress and other stress with sleep.

Heart Lung 2001;30:237-43.

33. Rhudy JL, Meagher MW. Noise stress and human pain thresholds: Divergent effects in men and women. J Pain 2001;2:57-64.

34. Haskard KB, Williams SL, DiMatteo MR, Heritage J, Rosenthal R. The provider's voice: Patient satisfaction and the content-filtered speech of nurses and physicians in primary medical care. J Nonverbal Behav 2008;32:1-20.

35. Hall JA, Irish JT, Roter DL, Ehrlich CM, Miller LH. Satisfaction, gender, and communication in medical visits. Med Care 1994;32:1216-31.

36. Watkins CD, Fraccaro PJ, Smith FG, et al. Taller men are less sensitive to cues of dominance in other men. Behav Ecol 2010;21:943-7.

37. von Baeyer CL, Piira T, Chambers CT, Trapanotto M, Zeltzer LK. Guidelines for the cold pressor task as an experimental pain stimulus for use with children. J Pain 2005;6:218-27.

38. Mitchell LA, MacDonald RR, Brodie EE. Temperature and the cold pressor test. J Pain 2004;5:233-8.

39. Cohen J. Statistical Power Analysis for the Behavioral Sciences, 2nd edn. Hillsdale: Lawrence Erlbaum, 1988.

40. Pud D, Golan Y, Pesta R. Hand dominancy - a feature affecting sensitivity to pain. Neurosci Let 2009;467:237-40.

41. Vigil JM, Rowell LN, Maestes R. Laboratory personnel gender and cold pressor apparatus affect subjective pain reports. Pain Res Manag 2014;19:e13-e19. 


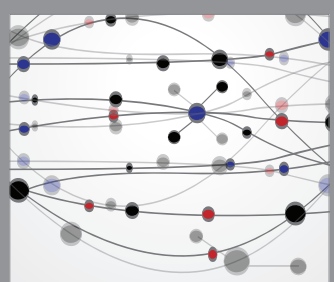

The Scientific World Journal
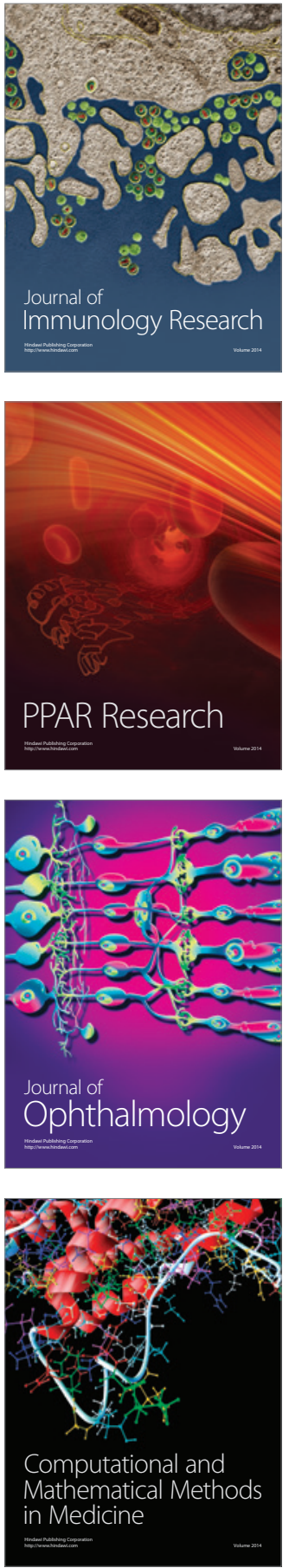

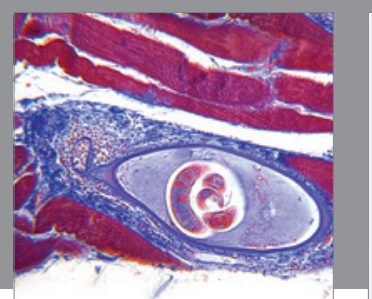

Gastroenterology Research and Practice

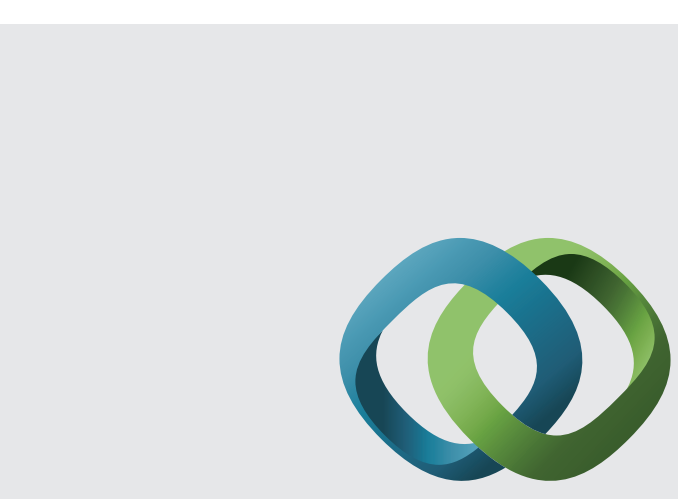

\section{Hindawi}

Submit your manuscripts at

http://www.hindawi.com
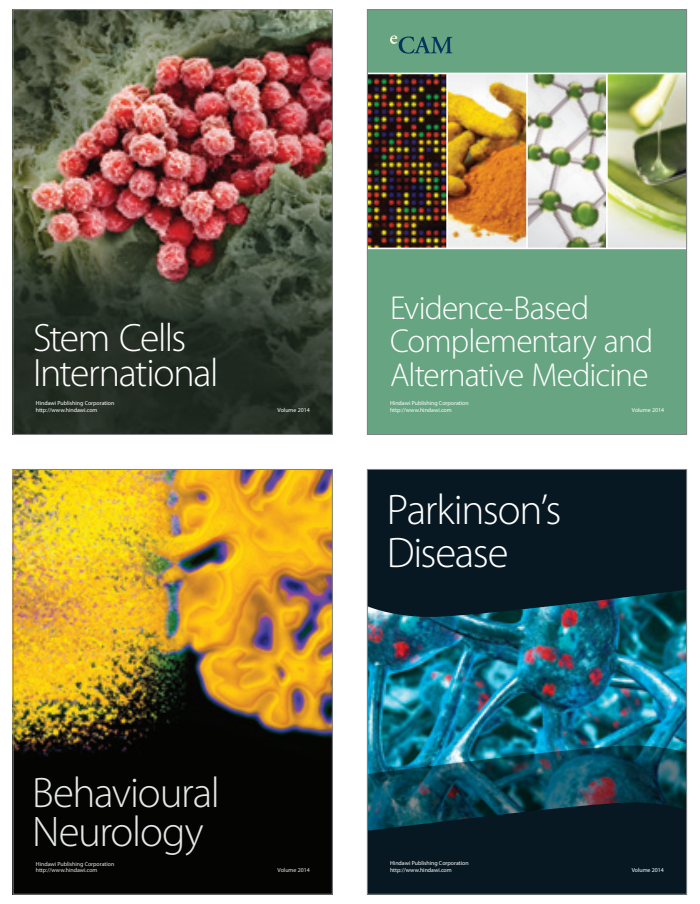
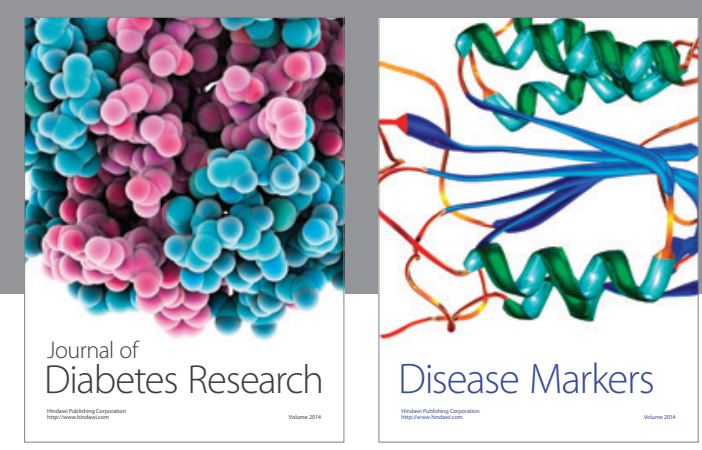

Disease Markers
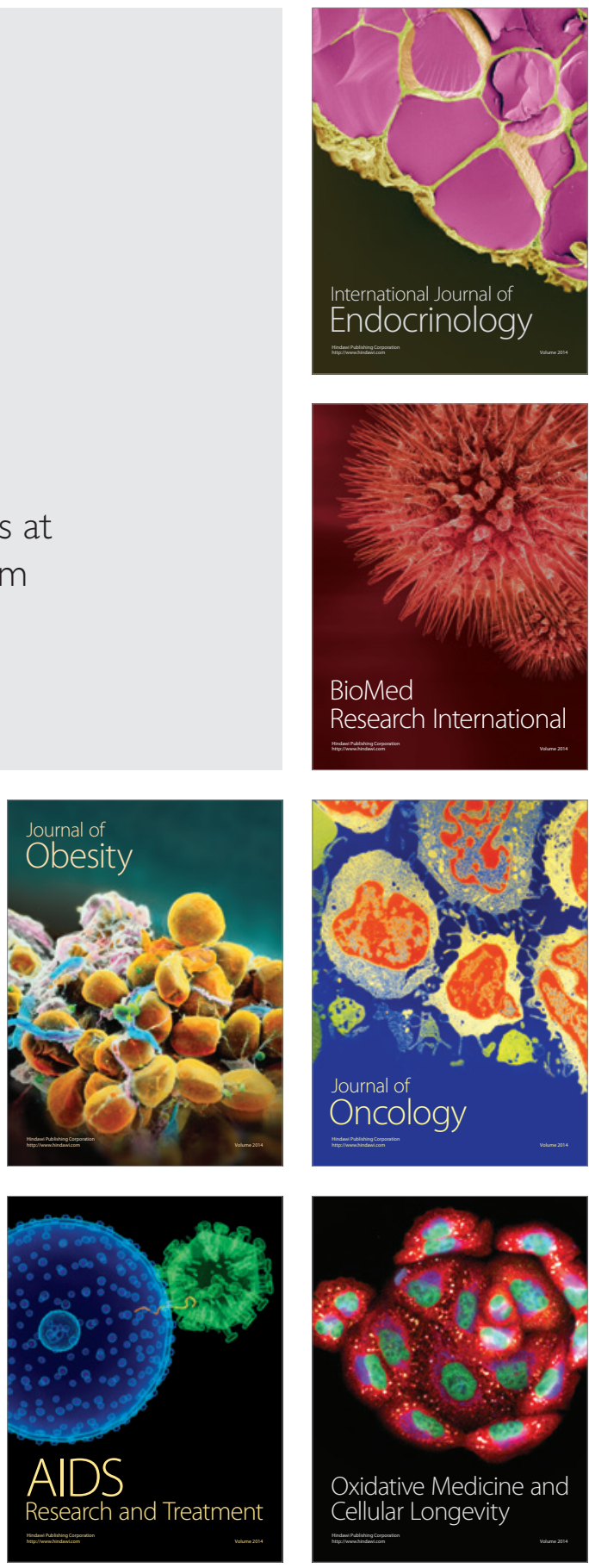